\title{
A New Image Quality Metric using System Visual Human Characteristics
}

\author{
Habiba Loukil \\ Science and \\ ImageTechnologies and \\ Telecommunications \\ (SETIT Sfax) Tunisia
}

\author{
Moez Hadj Kacem \\ Laboratory of Electronic and \\ Information Technology \\ (LETI-Sfax) Tunisia
}

\author{
Mohamed Salim BouhleL \\ Science and Image \\ Technologies and \\ Telecommunications \\ (SETIT Sfax) Tunisia
}

\begin{abstract}
Several human visual system (HVS) based quality metrics have been developed in recent years to measure the quality distortions caused by digital image coding techniques. Because of the complicated nature of the HVS characteristics, these metrics do not provide acceptable correlation with perceptual evaluations of the distortion. Recent studies by the Visual Quality Experts Group (VQEG) also show that the current HVS-based techniques do not provide a clear advantage over a mathematically defined technique such as mean square error (MSE). Therefore, this paper proposes a new quality metric based on the dynamic characteristics and on the just-noticeable-difference threshold characteristics introduced by Weber's law: the relative weighted Peak to Signal Noise Ratio. Our experiments on various image distortion types indicate that our metric performs significantly better than the widely used distortion metric Mean Squared Error. We should that the rwPSNR correlates with human perception of image quality.
\end{abstract}

\section{Keywords}

Weber's law, human visual system (HVS), means squared error (MSE), rwPSNR.

\section{INTRODUCTION}

Image quality measures play important roles in various image processing applications[1]. There are basically two distortion assessment approaches: objective measures and subjective measures. Subjective assessment tests are widely used to evaluate the image quality, but they are difficult and lengthy and results obtained might be very depending on the test condition and human viewers [1]. Therefore, they require longer time and the results are not always repeatable. Objective quality metrics are error-based methods. Thus, these mathematical measures are performed by pixel based difference metrics like MSE, Root MSE (RMSE), Mean Absolute Errors (MAE), and Signal to Noise Ratio (SNR) and Peak Signal to Noise Ratio (PSNR). However, these metrics do not correlate with the perceived image quality[2]. Indeed, they neglect the properties of the HVS and thus cannot be a reliable predictor of the perceived visual quality.

In the last decade, several image and video quality metrics have been proposed, which incorporate perceptual quality measures by considering the HVS characteristics [1,2]. Unfortunality, none of these uncomplicated objective metrics in the literature has shown any clear advantage over simple mathematical measures such as PSNR under strict testingconditions and different image distortion environments [3], [4], [5].
Mathematically defined measures are still attractive because of two reasons. First, they are easy to calculate and usually have low computational complexity. Second, they are independent of viewing conditions and individual observers. Although it is believed that the viewing conditions play important roles in human perception of image quality, they are, in most cases, not fixed and specific data is generally unavailable to the image analysis system. If there are $\mathrm{N}$ different viewing conditions, a viewing condition dependent method will generate $\mathrm{N}$ different measurement results that are inconvenient to use. In addition, it becomes the user's responsibilities to measure the viewing condition and to calculate and input the condition parameters to the measurement systems. By contrast, a viewing conditionindependent measure delivers a single quality value that gives a general idea of how good the image is.

In this paper, we propose a new image quality metric which is easy to calculate and applicable to various image processing applications. Instead of using traditional error summation methods, the proposed metric is designed by modeling any image distortion.

More importantly, our new metric is applicable to various image processing applications and provide meaningful comparison across different types of image distortion.

\section{WEBER'S LAW OF JUST NOTICEABLE DIFFERENCES}

This law hails from the middle of the nineteenth century. It is mainly founded on experiments where persons were given two nearly identical stimuli and tested whether they could notice a difference between them. It was found that the smallest noticeable difference was roughly proportional to the intensity of the stimulus. For example: Suppose that you presented two spots of light each with an intensity of 100 units to an observer. Then you asked the observer to increase the intensity of one of the spots until it was just noticeably brighter than the other. If the brightness needed to yield the just noticeable difference was 110 then the observer's difference threshold would be 10 units (i.e., delta $\mathrm{I}=110$ - 100 $=10)$. The Weber fraction equivalent for this difference threshold would be 0.1 (delta $\mathrm{I} / \mathrm{I}=10 / 100=0.1$ ).

It is important in understanding these effects to define a new metric which respect this law.

\section{DEFINITION OF THE NEW QUALITY INDEX}

Although the PSNR quantifies the intensity of the distortion, it doesn't adjust to the dynamic characteristics of the image. Indeed the deterioration is more visible in zones few textured (to weak variance) and less visible in zones more textured (stronger variance) [6]. Of this fact, we took account of the 
variance of the picture. It increases when the variance is big and decreases in the contrary case. We will have a new definition of the MSE.

Let $X=\left\{x_{i j} \mid I=1, . ., M ; j=1, . ., N\right\}$ and $Y=\left\{y_{i j} \mid I=1, ., M\right.$ $; j=1, \ldots, N\}$ be the original image and the test image, respectively. The wMSE is given as:

$$
w M S E=\frac{1}{M N} \sum_{m=0}^{M-1} \sum_{n=0}^{N-1}\left(\frac{\mid\left(x_{m, n}-y_{m, n}\right)}{1+\operatorname{Var}(M, N)}\right)^{2}
$$

Where, $\operatorname{Var}(\mathrm{M}, \mathrm{N})$ is the test image varianceIn the other hand, the sensitivity of the HVS to the errors may be different for different intensities. Therefore, human eyes haven't an equal sensitivity across different intensities. In fact, there was a threshold of sensitivity that must be passed before an increase of the intensity so that it can be detected. This is known as Weber's Law. The Weber's law introduces the concept of "just noticeable difference". So as complement to the wPSNR, we introduce our rwPSNR "relative weighted PSNR" that takes account of the relative difference of gray levels of the image [7] because the noticeable difference of two stimulus was roughly proportional to the intensity of the stimulus. Indeed, the difference of intensity (10) brings in two pixels of values respective 10 and 20 , is numerically the even that the one brings in a couple of pixels of values 110 and 120 . However on the visual plan the perception differs. It calls us therefore to think about the necessity to introduce the relative difference notion in the calculation of the wPSNR from where the rwPSNR. So we have a new definition of the MSE noted rwMSE "relative weighted Mean Square Error" which takes account of the variance and the intensity of the image. Our rwMSE is defined in this case:

Let $X=\{x \mid i=1, \ldots, M ; j=1, \ldots, N\}$ and $Y=\{y \mid i=1, \ldots, M$;

$\mathrm{j}=1, \ldots, \mathrm{N}\}$ be the original image and the test image,

respectively. The rwMSE is given as:

$r w M S E=\frac{1}{M N} \sum_{m=0}^{M-1} \sum_{n=0}^{N-1}\left(2 * \frac{|(x-y) /(x+y)|}{1+\operatorname{Var}(M, N)}\right)^{2}$

So the expression of our relative weighted peak signal to noise ratio is given by:

$$
r w P S N R=10 \log _{10} \frac{x_{\max }^{2}}{r w M S E}(3)
$$

\section{DEFINITION APPLICATION TO IMAGES}

Image signals are generally non-stationary while image quality is often also space variant, although in practice it is usually desired to evaluate an entire image using a single overall quality value.

We use images with different types of distortions to test our new quality metric and compare the results with the MSE and with subjective evaluations. The test images are distorted by a wide variety of corruptions: impulsive salt-pepper noise, additive Gaussien speckle noise, contrast streatching, JPEG compression... Some sample images are schown in figure 1 and 2, where we turned all the distortions to yield the same MSE relative to the original image. Our overall quality metric rwPSNR of each image is also calculated. In the subjective experiments, we present the original image and the distorted images.

In this experiment, the performance of MSE is extremely poor in the sense that images with nearly identically MSE are drastically different in perceived quality. By contrast, our new quality metric rwPSNR exhibits very consistent correlation with the subjective measures.

\section{RESULTS AND DISCUSSION}

To evaluate the performance of the proposed metric, we use images with different distortions [8]. Six image quality assessments are being compared, including MSE, and the proposed rwPSNR. Each set of images has, nearly, identical MSE. Objective image quality measures are calculated for all images and presented in figure1. The images with similar MSE have significantly different visual quality and rwPSNR delivers better consistency with perceptual valuations. Distortion measure for image should reflect the limitations of the HVS. In Figure 1, the image is created by adding a Gaussian noise, the image has a Gaussian noise and the image has a stretching contrast. The three images have got the same MSE. It is clear to note that those images have differentperceptual valuations. Our proposed metric outperforms significantly MSE in all cases, for both subjective and objective evaluations. With reference to the original Lena image The images in figure2 present a higher difference in terms of rwPSNR. But, these images do not show any Significant difference in terms of MSE. Therefore, the rwPSNR is more significant and competent then MSE(Figure1 and 2). So, this new metric is a better indicator of perceived image quality than the MSE. 


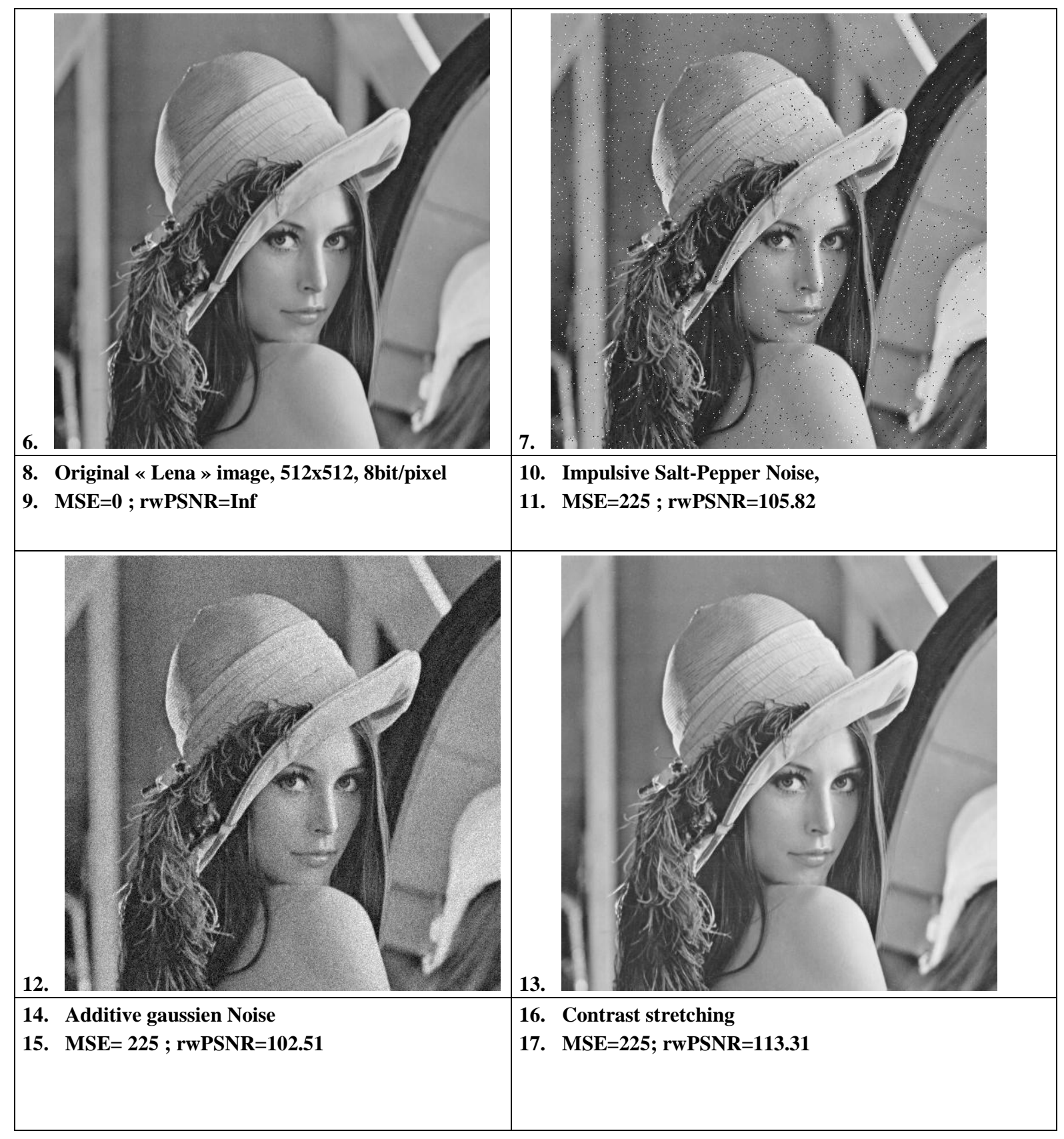

Fig 1: Lena with different types of distortions.

All distorted images have nearly identical MSE, but with drastically different visual quality. 

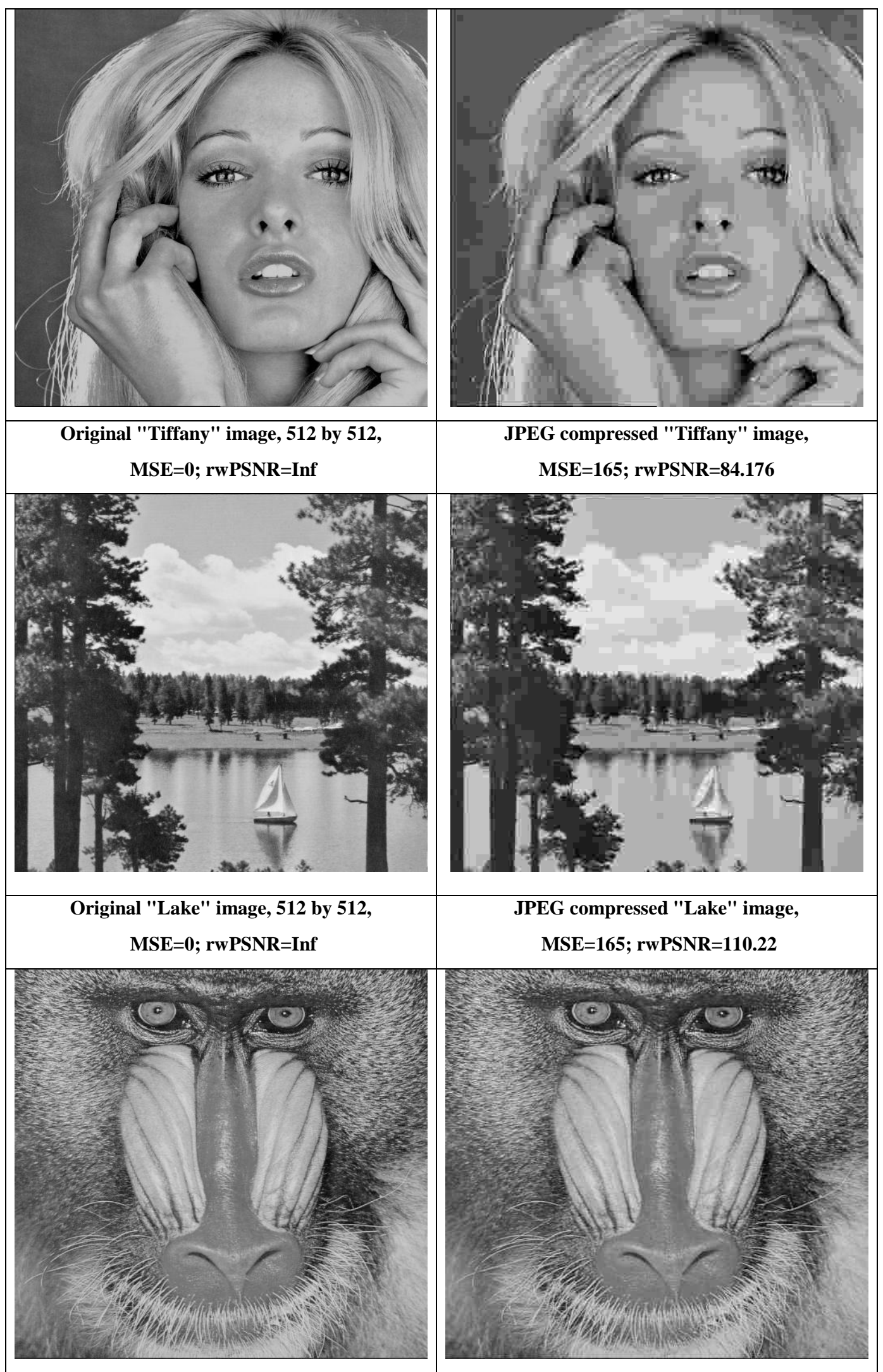

Original "Mandrill" image, 512 by 512, MSE=0; rwPSNR=Inf

JPEG compressed "Mandrill" image,

MSE=165; rwPSNR=129.74

Figure 2- Tiffany, Lakes and Mandrill with JPEG compression

All compressed images have similar MSE, but with very different visual quality 


\section{CONCLUSION}

A new image quality metric based on the relative difference of gray levels of the image introduced by Weber's law was proposed. Our experimental results on images with different types of distortion indicate that our new metric outperforms the MSE significantly under different types of image distortions. We think the success is due to its strong ability in measuring structural distortion occurred during the image degradation process. This is a clear distinction with MSE, which is sensitive to the energy of errors, instead of structural distortions. In the future, more extensive experiments are needed to fully validate our new metric.

There is no doubt that more precise modeling of the HVS is always advantageous in the design of image quality metrics. However, without a well-defined mathematical framework, the efforts in HVS modeling will not result in a successful quality measure.

\section{REFERENCES}

[1] T. N. Pappas, R. J. Safranek, and J. Chen, "Perceptual criteria for image quality evaluation," in Handbook of Image and Video Processing (A. C. Bovik, ed.), p. 939959, Academic Press, 2005.

[2] Shan Suthaharan, Seong-Whan Kim, and K.R. Rao. "A new quality metric based on just-noticeable difference, perceptual regions, edge extraction and human vision". EJ.-B. Martens and L.Meesters, "Image dissimilarity", Signal Processing, vol.70, pp.155-176, (Nov.1998).
[3] J.-B. Martens and L.Meesters, "Image dissimilarity", Signal Processing, vol.70, pp.155-176, (Nov.1998).

[4] W .JavedAamir, K .Khurram,S.Muhammad and A.Faisal Munir «Analyse de codage vidéo et outils de résilience aux erreurs de H. 264/AVC dans l'environnement sans fil » ;IJCA Journal ,Volume $50 \mathrm{~N}^{\circ} 1.2012$

[5] A.M.Eskicioglu and P.S. Fisher, "Image quality measures and their performance", IEEE Trans. Communications, vol.43, pp.2959-2965 (Dec.1995).

[6] H.Trichili, M.S.BOUHLEL, L.Kamoun, "Contribution aux Mesures Efficaces de l'Imperceptibilité du Tatouage et Nécessité de l'Introduction d'un masque psychovisuel", Second Scientific Young Researcherdays in Electric Genius and Data processing GEI'2002. Hammamet, Tunisia, pp.25-27 (March 2002)

[7] M.J.Nadenau, S. Winkler, D. Alleysson, and M. Kunt., "Humain Vision Models for perceptually optimized image processing- a review",Proc of the IEEE (Sept. 2000) Tavel, P. 2007 Modeling and Simulation Design. AK Peters Ltd.

[8] Wang, Z., A.C. Bovik, H.R. Sheikh and E.P. Simoncelli, "Image quality assessment: From error visibility to structural similarity". IEEE Trans. Image Processing, Vol: 13. (2004)Forman, G. 2003. An extensive empirical study of feature selection metrics for text classification. J. Mach. Learn. Res. 3 (Mar. 2003), 1289-1305. 